Ann. Sci. forest., 1980, 37 (1), 53-71.

\title{
Interception des précipitations et apport au sol d'éléments minéraux par les eaux de pluie ef les pluviolessivats dans une hêtraie atlantique et dans quelques peuplements résineux en Bretagne
}

\author{
F. FORGEARD, J. C. GLOAGUEN et J. TOUFFET
}

Laboratoire d'Ecologie végéfale,

Complexe scientifique de Beoulieu, 35042 Rennes Cedex

\section{Résumé}

L'interception des précipitations et l'apport au sol d'éléments minéraux par les eaux de pluie et les pluviolessivats ont éfé déferminés dans huif peuplements de résineux et dans une vieille futaie de Hêtres en 1973,1974 et 1975.

L'étude de l'interception a été poursuivie en 1976 et 1977 dans sepł stades de développement de la hêtraie.

\section{I. - Introduction}

L'étude de l'interception des précipitations par le couvert végétal a déjà fait l'objet de nombreux travaux, surtout en ce qui concerne les écosystèmes forestiers.

En Belgique, Noirfalise (1959) met en évidence un taux moyen d'interception de la pluie par le couvert végétal plus élevé pour les peuplements de résineux que pour les forêts feuillues. Cet auteur remarque également « un écoulement de la pluie le long des troncs beaucoup plus marqué pour les feuillus que pour les résineux, ce qui a pour conséquence d'amener plus d'eau à la sphère radiculaire des feuillus qu'à celle des résineux $\gg$.

Aussenac (1968) rappelle les hypothèses selon lesquelles l'eau interceptée pourrait être absorbée par l'écorce ou par les feuilles et ainsi améliorer le rendement photosynthétique des arbres ef modifier le climat (l'évaporation de l'eau interceptée correspond à une consommation d'énergie qui se traduit par un abaissement de température de l'air).

Ovington (1954), Lawson (1967), Schnock et Galoux (1967), White et Carlisle (1968) constatent que l'interception de l'eau de pluie par la couverture végétale varie selon l'espèce considérée, la structure du peuplement, l'intensité et la durée des précipitations. 
Une formulation mathématique de l'interception est également proposée par de nombreux auteurs : Hoover (1953), Skau (1964), Helvey et Patric (1965), Léonard (1966), Rapp ef Romane (1968), Rogerson et Byrnes (1968). Wolaver ef Lieth (1973) ont même proposé des modèles quantitatifs sur la composition minérale des eaux.

Les éléments minéraux arrivant au sol, par les eaux de pluie et les eaux de pluviolessivage représentent un apport important qui ne peut être négligé dans l'étude du cycle biogéochimique d'un écosystème. Miller (1963), Carlisle, Brown ef White (1966 et 1967), Denaeyer-de Smet (1966), Rapp (1969 et 1973), Nihlgard (1970), Aussenac, Bonneau et Le Tacon (1972), Gratani et Bruno (1974), Mayer et Ulrich (1974), Szabo et Csortos (1975) constatent que les apports en éléments biogènes sont extrêmement variables. II ressort de ces divers travaux que la composition de l'eau de pluie incidente est fonction de la distance à la mer et que celle des lessivats varie avec la nature du peuplement végétal et la période de l'année.

Notre étude a pour but de comparer, en climat atlantique, l'intercepíion des pluies et l'apport au sol d'éléments biogènes d'une part dans divers siades de développement d'une hêtraie et d'autre part dans des peuplements résineux de substitution. Elle a été effectuée au cours des années 1973 à 1977 en forêł de Villecartier et à quelques kilomètres au Sud-Ouest de cette forêt au lieu-dit le Bois Robert, sur la commune de Bazouges-la-Pérouse (Ille-et-Vilaine).

\section{II. - Stations étudiées. Méthodologie}

Les caractéristiques générales de la forêt onł déjà été exposées tant en ce qui concerne la végétation que les conditions écologiques (Touffet, 1964 et 1971 ; Esteoule, Guyader et Touffet, 1971). Un certain nombre de données concernant les peuplements étudiés (Gloaguen et Touffet, 1974 et 1976) sont regroupées dans le tableau 1.

L'égouttement a été suivi au cours des années 1973, 1974 ef 1975 dans des peuplements de Pinus sylvestris, Pinus nigra laricio, Pinus murrayana, Picea sitchensis, Picea abies, Tsuga heterophylla et $P$ seudotsuga menziesii et au cours des années 1974 et 1975 dans le peuplement d'Abies grandis. Dans la hêtraie il a été étudié en 1973, 1974 et 1975 dans une vieille futaie et en 1976 et 1977 dans sept peuplements d'âges différents : fourré, gaulis, jeune perchis, haut perchis, jeune futaie, moyenne futaie et vieille futaie.

Les pluviomètres sont constitués d'une bouteille en matière plastique, dont la partie supérieure est coupée et retournée pour former un entonnoir (d'une section de $56,35 \mathrm{~cm}^{2}$ ) et éviter l'évaporation. Le goulot est muni d'un filtre en nylon. Dix pluviomètres sont installés par station, à des emplacements fixes, sur deux lignes perpendiculaires orientées Nord-Sud et Ouest-Est ; espacés de $2 \mathrm{~m}$, légèrement enfoncés dans le sol, leur surface réceptrice est située à $20 \mathrm{~cm}$ de hauteur. En 1976 ef 1977 dans tous les peuplements de la hêtraie, le nombre de pluviomètres a été doublé. Une enclave non boisée a servi de station témoin « sol nu », avec dix pluviomètres en 1973, 1974 et 1975 et 20 en 1976 et 1977.

Chaque mois les volumes d'eau sont mesurés au laboratoire, à l'éprouvette. Pour tous les peuplements de résineux et pour la vieille futaie, les analyses chimiques ont été effectuées sur une partie aliquote d'eau filtrée en 1973, 1974 et 1975 suivanf les 
techniques déjà utilisées pour le dosage des eaux de pluie recueillies sous les landes (Forgeard, Gloaguen et Touffet, 1979).

L'écoulement le long des troncs n'a pas été mesuré. Pour compléter l'apport au sol d'éléments minéraux par le pluviolessivage, il a été estimé, pour chaque espèce, d'après les données bibliographiques.

TABLEAU 1

Principales caractéristiques des peuplements éfudiés

Main characteristics of the studied stands

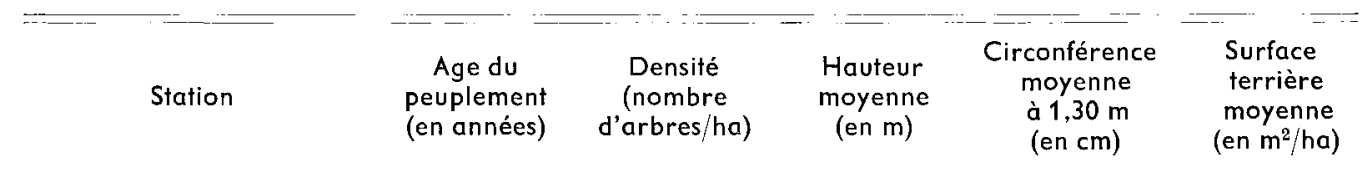

Hêtraie

\begin{tabular}{|c|c|c|c|c|c|}
\hline Fourré $\ldots \ldots \ldots \ldots \ldots$ & 10 & 60000 & 3 & 7 & 23 \\
\hline Gaulis $\ldots \ldots \ldots \ldots$. & $20-30$ & 32400 & 10 & 11 & 31 \\
\hline Bas Perchis ......... & $40-50$ & 4900 & 16 & 25 & 24 \\
\hline Haut Perchis ........ & $50-60$ & 2700 & 18 & 30 & 19 \\
\hline Jeune futaie. . ......... & $70-80$ & 800 & 27 & 77 & 38 \\
\hline Moyenne futaie........ & $110-120$ & 300 & 27 & 114 & 31 \\
\hline Vieille futaie . ......... & $120-140$ & 200 & 28 & 138 & 30 \\
\hline
\end{tabular}

Résineux

\begin{tabular}{|c|c|c|c|c|}
\hline Pinus sylvestris $\ldots \ldots \ldots$ & 20 & 2190 & 13 & 40 \\
\hline Pinus nigra laricio . . . . . . & 20 & 1850 & 12 & 40 \\
\hline Pinus murrayana . . . . . . & 20 & 1750 & 13 & 55 \\
\hline Picea sitchensis $\ldots \ldots \ldots$ & 20 & 2580 & 12 & 48 \\
\hline Piced abies $\ldots \ldots \ldots \ldots$ & 33 & 1280 & 20 & 59 \\
\hline Tsuga heterophylla....... & 24 & 1830 & 15 & 57 \\
\hline Pseudotsuga menziesii ... & 20 & 2530 & 13 & 42 \\
\hline Abies grandis . . . . . . . . & 20 & 2290 & 13 & 48 \\
\hline
\end{tabular}

\section{III. - Résultats}

1. - Pluviométrie et interception par le couvert végétal

\subsection{Pluviométrie.}

Les résultats que nous avons obtenus en station "sol nu » sont très proches de ceux de la station météorologique d'Antrain-sur-Covesnon (35). Sur 5 années de mesures la pluviosité moyenne est de $825,6 \mathrm{~mm}$ à Villecartier et de $816,4 \mathrm{~mm}$ à Antrain. Nous observons donc un excès de 1,1 p. 100 par rapport à la station d'Antrain.

L'erreur standard moyenne, exprimée en pourcentage de la moyenne esł de 2,9 p. 100 en station sol nu. Sous la hêtraie elle est dans les différents peuplements de : 5,0 p. 100 dans le fourré, 4,1 p. 100 dans le gaulis, 5,3 p. 100 dans le perchis, 4,1 p. 100 


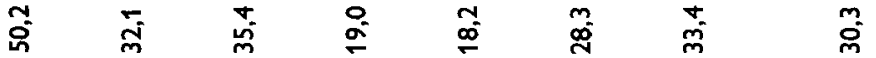

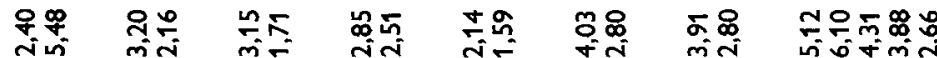

$\mathrm{HH} H \mathrm{H} H \mathrm{H} H \mathrm{H} H \mathrm{H} H+\mathrm{H} H+\mathrm{H} H+H+$

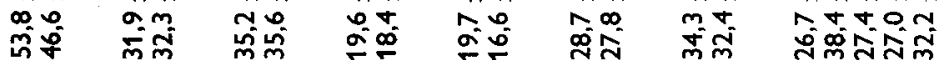

চi

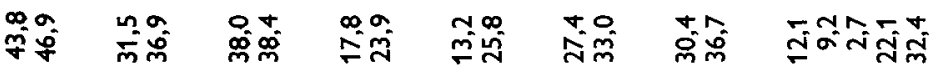

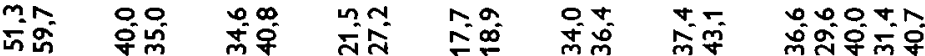

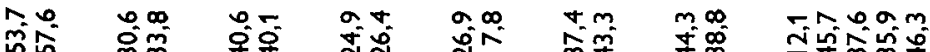

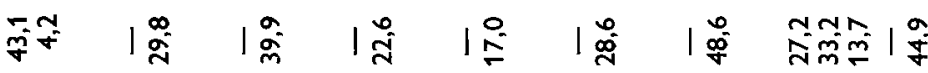

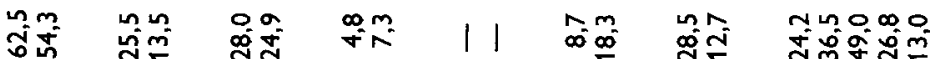

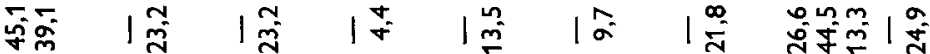

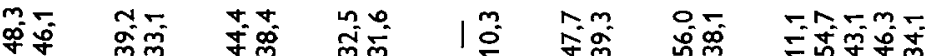

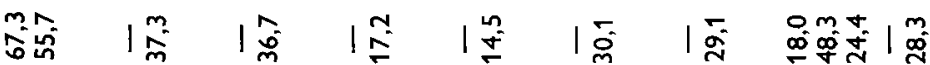
苛它 |

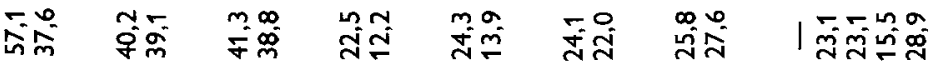

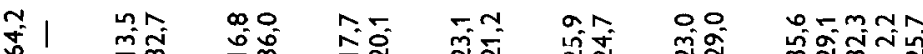

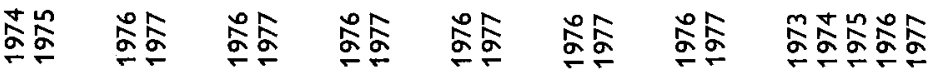
- 1 - 1 -

ㅎํำ

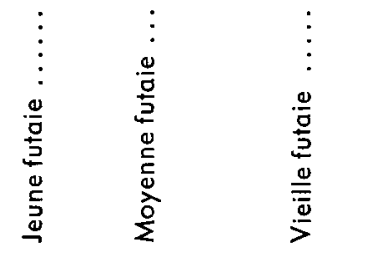

DכIIDAN/S sn60y 
dans le haut perchis, 3,3 p. 100 dans la jeune futaie, 3,1 p. 100 dans la moyenne futaie et 4,1 p. 100 dans la vieille futaie.

Sous les peuplements de Conifères cette erreur prend les valeurs suivantes : 3,6 p. 100 sous Pinus sylvestris, 4,8 p. 100 sous Pseudotsuga menziesii, 7,6 p. 100 sous Pinus murrayana, 8,2 p. 100 sous Pinus nigra laricio, 8,8 p. 100 sous Abies grandis, 9,0 p. 100 sous Tsuga heterophylla, 13,8 p. 100 sous Picea abies ef 15,2 p. 100 sous Picea sitchensis.

L'erreur standard présente de plus grandes variations sous les résineux (de 3,6 à 15,2 p. 100) que dans les différentes parcelles de la hêtraie (de 3,1 à 5,3 p. 100). Elle est indépendante de l'âge des peuplements et semble en relations avec la densité et l'homogénéité des frondaisons qui varient selon les espèces.

\subsection{Interception par le couvert végétal (tabl. 2).}

En ne prenant en compte que les eaux d'égouttement, l'interception moyenne annuelle des eaux de pluie par les différents peuplements varie de 18,2 p. 100 à 35,4 p. 100 sous la hêtrale et de 42,6 p. 100 à 60,2 p. 100 sous les Conifères.

Les relations entre le pourcentage d'interception et la surface terrière des différents peuplements sont représentées sur la figure 1 : pour une même surface terrière, les Conifères ont toujours une interception supérieure à celle du Hêtre. Pour celui-ci, l'interception, bien qu'assez variable, semble indépendante de l'âge du peuplement ; elle dépend plutôt de sa structure : ainsi elle est faible dans les deux perchis qui viennent d'être éclaircis.

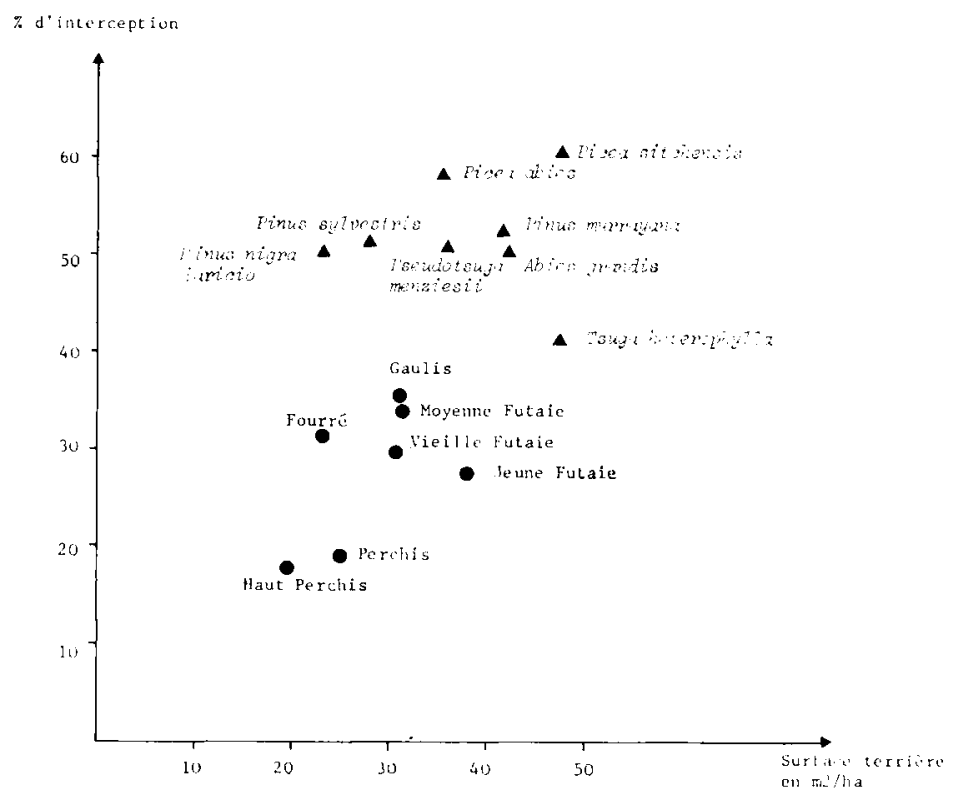

FIG. 1. - Relations entre le pourcentage d'interception et la surface terrière des différents peuplements. Relations between percentage of interception and basal area of different stands. 
Si nous nous référons aux travaux de Horton (1919), Ovington (1954), Noirfalise (1959), Aussenac (1968 et 1975), Nihlgard (1970), Rapp ef Ibrahim (1978) et Boulangeat (1978) (tabl. 3), nos résultats sont du même ordre de grandeur que les leurs sous la hêtraie. Par contre sous Conifères nos valeurs sont généralement supérieures à celles de ces auteurs. Ceci s'explique par le fait que les peuplements que nous avons étudiés sont jeunes et très denses.

\section{TABLEAU 3}

Pourcentage d'interception dans divers peuplements (données bibliographiques)

Percentage of inferception in various stands (bibliographical data)

\begin{tabular}{|c|c|c|c|c|}
\hline Auteur & Espèce & Interception & Egouttement & Ecoulement \\
\hline $\begin{array}{l}\text { Noirfalise, } 1959 \\
\text { Nihlgard, } 1970 \\
\text { Aussenac, } 1968\end{array}$ & $\begin{array}{l}\text { Fagus sylvatica } \ldots \ldots \ldots \\
\text { Fagus sylvatica } \ldots \ldots \ldots \\
\text { Fagus sylvatica } \ldots \ldots \ldots \\
\text { Carpinus befulus } \ldots \ldots \ldots\end{array}$ & $\begin{array}{l}25 \text { p. } 100 \\
16,9 \text { p. } 100\end{array}$ & $\begin{array}{l}68,7-70,9 \text { p. } 100 \\
76,0 \text { p. } 100\end{array}$ & $\begin{array}{c}11,4-11,5 \text { p. } 100 \\
7,1 \text { p. } 100\end{array}$ \\
\hline $\begin{array}{l}\text { Aussenac, } 1975 \\
\text { Horton, } 1919\end{array}$ & $\begin{array}{l}\text { Fagus sylvatica } \ldots \ldots \ldots \ldots \\
\text { Fagus sylvatica } \ldots \ldots \ldots\end{array}$ & $\begin{array}{c}23,8-28,0 \text { p. } 100 \\
21,8 \text { p. } 100\end{array}$ & $\begin{array}{c}56,9-66,9 \text { p. } 100 \\
65,4 \text { p. } 100\end{array}$ & $\begin{array}{l}9,2-15,0 \text { p. } 100 \\
12,8 \text { p. } 100\end{array}$ \\
\hline $\begin{array}{l}\text { Noirfalise, } 1959 \\
\text { Nihlgard, } 1970 \\
\text { Aussenac, } 1968 \\
\text { Horton, } 1919 \\
\text { Ovington, } 1954\end{array}$ & $\begin{array}{l}\text { Picea abies } \ldots \ldots \ldots \ldots \\
\text { Picea abies } \ldots \ldots \ldots \ldots \\
\text { Picea abies } \ldots \ldots \ldots \\
\text { Picea abies } \ldots \ldots \ldots \\
\text { Picea abies } \ldots \ldots \ldots \\
\text { Pick }\end{array}$ & $\begin{array}{c}23-38 \text { p. } 100 \\
\begin{array}{c}34,5-34,3 \text { p. } 100 \\
58,8 \text { p. } 100\end{array}\end{array}$ & $\begin{array}{l}57,5-58,2 \text { p. } 100 \\
63,6-63,9 \text { p. } 100 \\
39,8 \text { p. } 100 \\
52 \text { p. } 100\end{array}$ & $\begin{array}{l}2,6-4,2 \text { p. } 100 \\
1,9-1,8 \text { p. } 100 \\
1,4 \text { p. } 100 \\
0,13 \text { p. } 100\end{array}$ \\
\hline $\begin{array}{l}\text { Noirfalise, } 1959 \\
\text { Aussenac, } 1968 \\
\text { Horton, } 1919 \\
\text { Ovington, } 1954 \\
\text { Rapp et lbrahim, } 1978\end{array}$ & $\begin{array}{l}\text { Pinède } \ldots \ldots \ldots \ldots \ldots \\
\text { Pinus syivestris } \ldots \ldots \ldots \ldots \\
\text { Pinus sp. } \ldots \ldots \ldots \ldots \ldots \\
\text { Pinus nigra } \ldots \ldots \ldots \ldots \ldots \\
\text { Pinus pinea } \ldots \ldots \ldots \ldots \ldots\end{array}$ & $\begin{array}{c}28 \text { p. } 100 \\
31,1-30,5 \text { p. } 100\end{array}$ & $\begin{array}{c}67,2-68 \text { p. } 100 \\
52,5 \text { p. } 100 \\
70,1 \text { p. } 100\end{array}$ & $\begin{array}{l}1,7-1,5 \text { p. } 100 \\
0-2,5 \text { p. } 100 \\
0,21 \text { p. } 100 \\
2,3 \text { p. } 100\end{array}$ \\
\hline $\begin{array}{l}\text { Ovington, } 1954 \\
\text { Ovington, } 1954 \\
\text { Aussenac, } 1968 \\
\text { Aussenac, } 1975 \\
\text { Boulangeat, } 1978\end{array}$ & $\begin{array}{l}\text { Tsuga sp. . . . . . . . . . . } \\
\text { Pseudotsuga taxifolia } \ldots \\
\text { Abies grandis. . . . . . . . . . } \\
\text { Abies grandis. . . . . . . . . } \\
\text { Pseudotsuga menziessii. . } \\
\text { Pseudotsuga menziessii. . }\end{array}$ & $\begin{array}{c}43,1-41,2 \text { p. } 100 \\
32,3-36,4 \text { p. } 100 \\
35-37 \text { p. } 100\end{array}$ & $\begin{array}{c}54 \text { p. } 100 \\
46 \text { p. } 100 \\
49 \text { p. } 100 \\
55,8-57,8 \text { p. } 100 \\
48,6-60,0 \text { p. } 100 \\
46-62 \text { p. } 100\end{array}$ & $\begin{array}{c}0,13 \text { p. } 100 \\
1,1-1,0 \text { p. } 100 \\
6,2-15,0 \text { p. } 100 \\
4-17 \text { p. } 100\end{array}$ \\
\hline
\end{tabular}

Alors que Aussenac (1968) sous Fagus sylvatica ef Carpinus betulus et Schnock et Galoux (1967) dans une chênaie mélangée ont mis en évidence une interception plus forte en période feuillée qu'en période défeuillée, Noirfalise (1959) trouve dans une chênaie à Bouleau un taux d'interception plus faible pendant l'été. Dans les peuplements de Fagus sylvatica que nous avons étudiés, il n'a pas été possible de mettre en évidence une relation entre les taux d'interception et les phénophases. Ceci peut s'expliquer par la nature des pluies; elles sont généralement plus violentes et ainsi moins facilement interceptées au printemps et en été, ce qui compense l'effet des feuilles. Par contre dans les peuplements de Conifères aù le feuillage est persistant, le taux d'interception est généralement plus important en automne-hiver, période où dominent les crachins. 


\section{2. - Composition minérale des eaux de pluie ef de pluviolessivage}

\subsection{Composition minérale des eaux de pluie.}

La composition minérale moyenne annuelle des eaux de pluie incidentes a été établie sur 3 ans ; elle est reportée dans le tableau 4 avec les valeurs obtenues à la même période à Fréhel et à Paimpont (Forgeard, Gloaguen et Touffet, 1979).

\section{TABLEAU 4}

Composition minérale des eaux de pluie incidentes (moyennes annuelles en ppm éfablies sur 3 ans) Mineral composition of rainfall (yearly means in ppm determined during three years)

\begin{tabular}{|c|c|c|c|c|c|c|}
\hline Station & $\begin{array}{c}\text { Distance à la mer } \\
\text { en } \mathrm{km}\end{array}$ & $\mathrm{Na}$ & $K$ & $\mathrm{Ca}$ & $\mathrm{Mg}$ & $N$ \\
\hline $\begin{array}{l}\text { Villecartier } \ldots \ldots \ldots \ldots \ldots \\
\text { Paimpont } \ldots \ldots \ldots \ldots \ldots \ldots \\
\text { Fréhel } \ldots \ldots \ldots \ldots \ldots \ldots\end{array}$ & $\begin{array}{r}20 \mathrm{~km} \\
70 \mathrm{~km} \\
<1 \mathrm{~km}\end{array}$ & $\begin{array}{r}2,59 \\
2,52 \\
17,57\end{array}$ & $\begin{array}{l}2,33 \\
1,65 \\
6,55\end{array}$ & $\begin{array}{l}1,12 \\
1,32 \\
2,44\end{array}$ & $\begin{array}{l}0,42 \\
0,43 \\
2,06\end{array}$ & $\begin{array}{l}1,44 \\
1,23 \\
1,84\end{array}$ \\
\hline
\end{tabular}

Pour tous les éléments, les valeurs à Fréhel sont supérieures à celles de Villecartier ef de Paimpont. Dans ces deux dernières stations les teneurs sont voisines en restant cependant légèrement plus fortes à Villecartier, plus proche de la mer $(20 \mathrm{~km})$, notamment pour le potassium et l'azote.

Comme Madgwick et Ovington (1959) nous constatons que le sodium est plus abondant en hiver et présente des teneurs plus faibles au printemps et en été (fig. 2) ; c'est ce que nous avions déjà constaté à Fréhel et à Paimpont. Par contre, pour les autres éléments, nous ne pouvons mettre en évidence de variations saisonnières. Les teneurs en potassium sont extrêmement variables d'un mois à l'autre alors que le taux de magnésium et de calcium ne subit pas de variations très importantes.

\subsection{Composition minérale des eaux d'égoutfement.}

2.21. - Teneurs moyennes annuelles.

Après passage sur le couvert forestier, les lessivats sont toujours plus concentrés que les eaux de pluie incidentes. En fonction des teneurs croissantes en éléments des eaux d'égouttement, les espèces sont ordonnées dans les cinq séries suivantes :

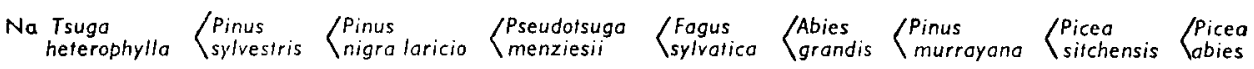

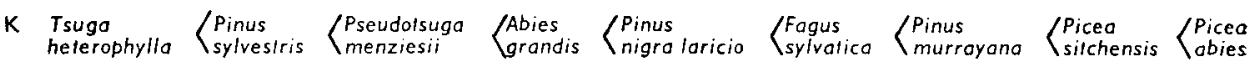

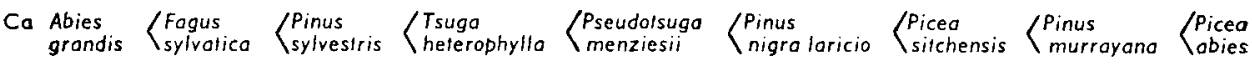

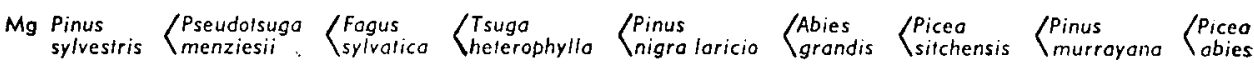

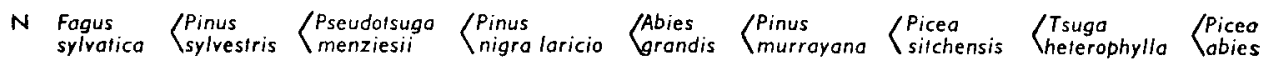




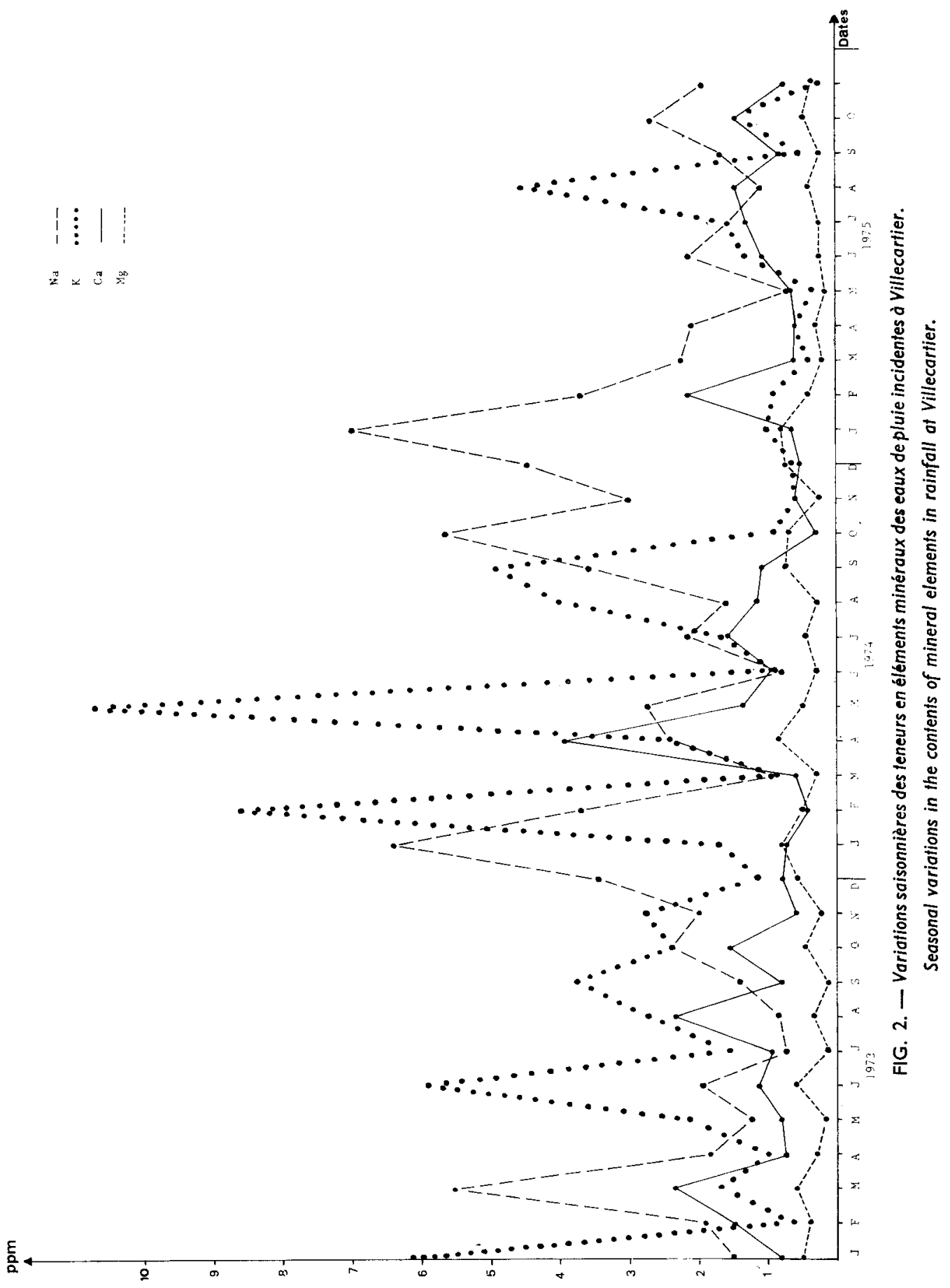


C'est toujours sous les boisements de Picea abies que les teneurs sont les plus élevées, puis généralement sous les peuplements de Pinus murrayana et de Picea sitchensis. Les eaux recueillies sous Pinus sylvestris sont au contraire peu concentrées. Pour d'autres espèces comme Tsuga heterophylla et Abies grandis, la position dans les séries est très variable suivant l'élément considéré. Le tableau 5 permet de comparer les teneurs en éléments des eaux d'égouttement avec celles des feuilles vertes et des litières arrivant au sol dans les divers peuplements.

C'est en potassium que les eaux de pluie s'enrichissent le plus par lessivage. Celui-ci provient surtout des feuilles, en effet leurs teneurs sont, pour toutes les espèces, nettement plus élevées lorsqu'elles sont vertes que l'orsqu'elles tombent à terre.

Inversement, l'enrichissement des eaux d'égouttement est toujours faible en calcium, élément plus concentré dans les feuilles sèches que dans les feuilles vertes.

Les feuilles vertes sont généralement plus riches en magnésium, élément qui est comme le potassium abondamment « recrété », alors que pour le sodium les résultats varient selon les espèces.

Les différences de concentration des lessivats observées d'un peuplement à l'autre ne dépendent donc pas uniquement de la composition des aiguilles mais de leur aptifude à « recréter » les éléments minéraux.

\subsection{Variations des teneurs mensuelles.}

- Sodium.

Les eaux de lessivage sont plus concentrées pendant la période hivernale que pendant l'été, que ce soit sous peuplements de feuillus ou de Conifères. Nous avions déjà observé ce phénomène dans les landes (Forgeard, Gloaguen et Touffet, 1979). Les valeurs maximales ont été observées en janvier 1975 et les minimales en juillet 1973. C'est sous les peuplements d'Epicéa que les écarts sont les plus importants, de $59 \mathrm{ppm}$ à 1,7 ppm pour Picea sitchensis et de $70 \mathrm{ppm}$ à 1,3 ppm pour Picea abies. Sous d'autres essences, les variations encore notables sont de moins grande intensité (de 15,8 ppm à 1,4 ppm pour Tsuga heterophylla, de 16,6 ppm à 1,5 ppm pour Pseudotsuga menziesii).

- Potassium.

Les teneurs en potassium présentent d'importantes mais irrégulières variations au cours de l'année. Il est difficile ici de mettre en évidence, ainsi que Lausberg l'avait observé en 1935, une période de récrétion en automne sous les feuillus. Les teneurs sont aussi variables d'une année à l'autre : en 1974 les valeurs sont supérieures à celles de 1973 et 1975.

\section{- Calcium el magnésium.}

Les teneurs en calcium des pluviolessivats présentent des valeurs minimales en période printemps-été et des valeurs maximales en période hivernale. En 1974, les concentrations élevées se sont prolongées jusqu'au début du printemps (avril-mai) après une baisse en février-mars, et les minima ont été décalés vers l'automne. Ainsi par exemple les valeurs maximales mesurées sous peuplement de Pinus murrayana sont de 13,4 ppm en mars 1973, 11,4 ppm en décembre 1973, 14,4 ppm en avril 1974, 8,2 ppm en décembre 1974, 10,3 ppm en décembre 1975 alors que pour cefte essence les minima sont de 1,5 ppm en septembre 1973 et 1975. 


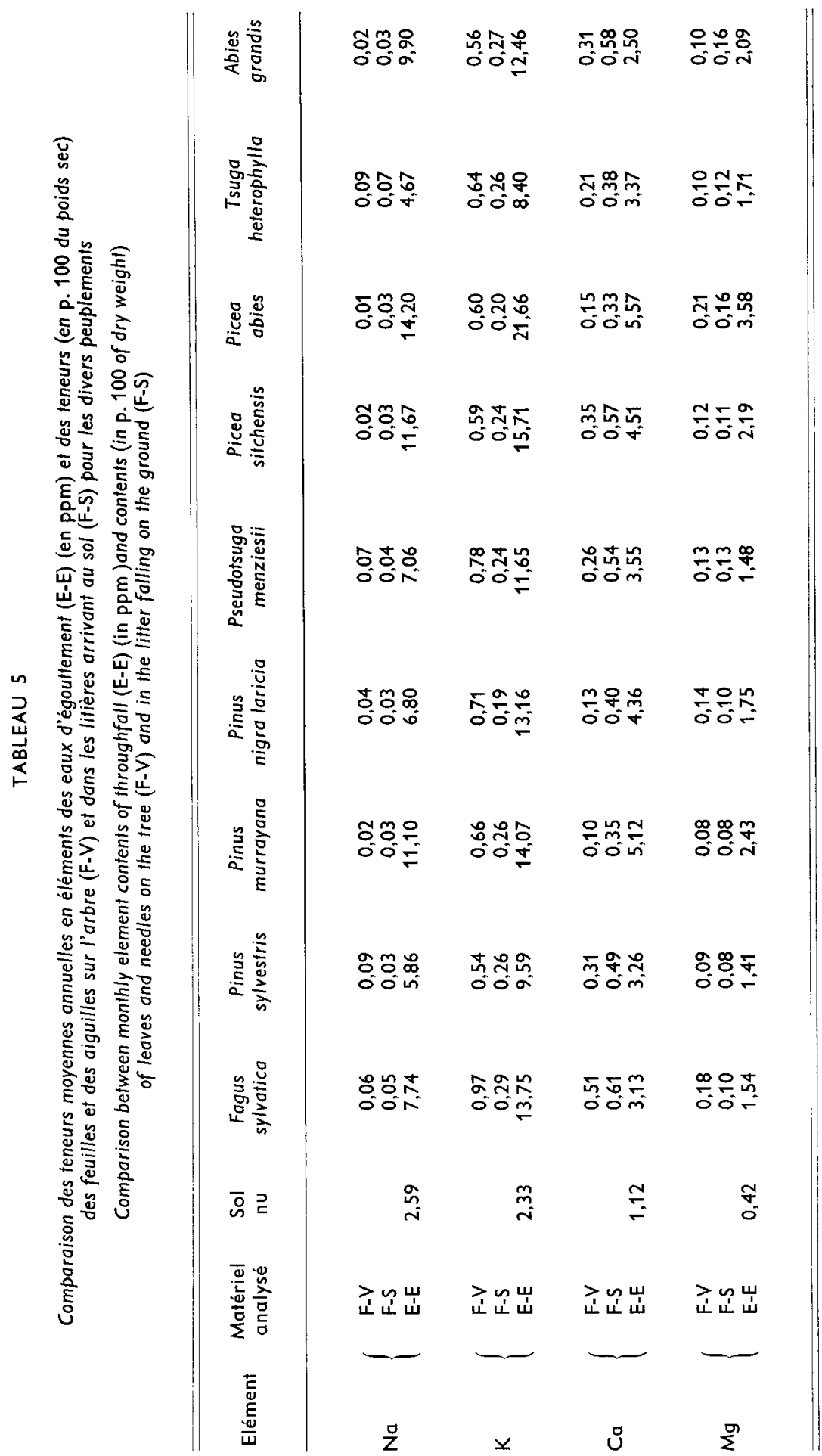


Les teneurs en magnésium subissent des variations comparables à celles que nous avons observées pour le calcium avec des valeurs minimales en été (depuis 0,4 ppm) et maximales en hiver (jusqu'à 11 ppm).

Denaeyer-de Smet (1966) avait déjà signalé des teneurs en calcium plus élevées au cours de l'hiver que pendant l'été tant dans les eaux incidentes que sous couvert forestier.

- Azote.

Les teneurs en azote subissent des variations irrégulières ; peu marquées dans la hêtraie (de 0,4 à 4 ppm), elles prennent une plus grande amplitude sous certaines essences résineuses comme Tsuga heterophylla (de 1,2 à 18,6 ppm) ou Picea abies (de 1,5 à 16,4 ppm).

\section{3. - Les apports d'éléments minéraux par les eaux de pluie et les lessivats}

\subsection{Par les eaux incidentes.}

Nous avions vu précédemment (Forgeard, Gloaguen et Touffet, 1979) que les apports annuels totaux $(\mathrm{Na}+\mathrm{K}+\mathrm{Ca}+\mathrm{Mg}+\mathrm{N})$ par les eaux de pluie atteignaient $52 \mathrm{~kg} / \mathrm{ha} / \mathrm{an}$ à Paimpont et $190 \mathrm{~kg} / \mathrm{ha} / \mathrm{an}$ à Fréhel et dépendaient donc essentiellement de la proximité de la mer. A Villecartier (tabl. 6), l'apport annuel total (moyenne effectuée sur 3 ans) est de $68,5 \mathrm{~kg} / \mathrm{ha} / \mathrm{an}$. L'élément le mieux représenté est le sodium $24,3 \mathrm{~kg} / \mathrm{ha} / \mathrm{an}$. Le potassium et l'azote sont également apportés en grande quantité $(19,0$ et $13,5 \mathrm{~kg} / \mathrm{ha} / \mathrm{an})$, le calcium ef le magnésium en quantités plus faibles ( 8 et $4 \mathrm{~kg} /$ ha/an).

\subsection{Par les pluviolessivats.}

\subsection{Par les eaux d'égoutfement.}

Les quantités d'éléments minéraux apportés par les eaux d'égouttement sous les différents peuplements étudiés sont reportées dans le tableau 6.

Les apports sous couvert forestier sont toujours supérieurs aux apports directs par les eaux de pluie incidentes. C'est sous la hêtraie que ces apports atteignent la plus grande valeur avec $146 \mathrm{~kg} / \mathrm{ha} / \mathrm{an}$. Sous les boisements de Conifères ils varient de $87,5 \mathrm{~kg} / \mathrm{ha} / \mathrm{an}$ (Pinus sy/vestris) à $138 \mathrm{~kg} / \mathrm{ha} / \mathrm{an}$ (Pinus murrayana). En fonction des apports totaux d'éléments minéraux par les eaux d'égouttement, les espèces peuvent être classées dans l'ordre suivant : Pinus sylvestris $(87,5 \mathrm{~kg} / \mathrm{ha} / \mathrm{an}<$ Pseudotsuga menziesii $(91,5 \mathrm{~kg})<$ Tsuga heterophylla $(100 \mathrm{~kg})<$ Picea sitchensis $(101 \mathrm{~kg})<$ Pinus nigra laricio $(114 \mathrm{~kg})<$ Abies grandis $(117,5 \mathrm{~kg})<$ Picea abies $(119 \mathrm{~kg})<$ Pinus murrayana $(138 \mathrm{~kg})<$ Fagus sylvatica $(146 \mathrm{~kg})$.

Alors que dans les eaux incidenies le sodium est l'élément dominant, dans les eaux d'égouttement le potassium est toujous plus abondant quel que soit le peuplement et représente un apport de 36 à $73,5 \mathrm{~kg} / \mathrm{ha} / \mathrm{an}$. Sous la hêtraie la quantité de potassium est nettement supérieure à celle des peuplements de Conifères. L'apport de sodium par les eaux d'égouttement varie de $22,5 \mathrm{~kg} / \mathrm{ha} /$ an pour Pinus sylvestris à $42,5 \mathrm{~kg} / \mathrm{ha} /$ an pour la hêtraie et pour Pinus murrayana.

Les apports en calcium el en azote sont moindres, ceux de calcium sont supérieurs à ceux d'azote sous Fagus sylvatica, Pinus sylvestris, Pinus nigra laricio, Pinus murrayana 


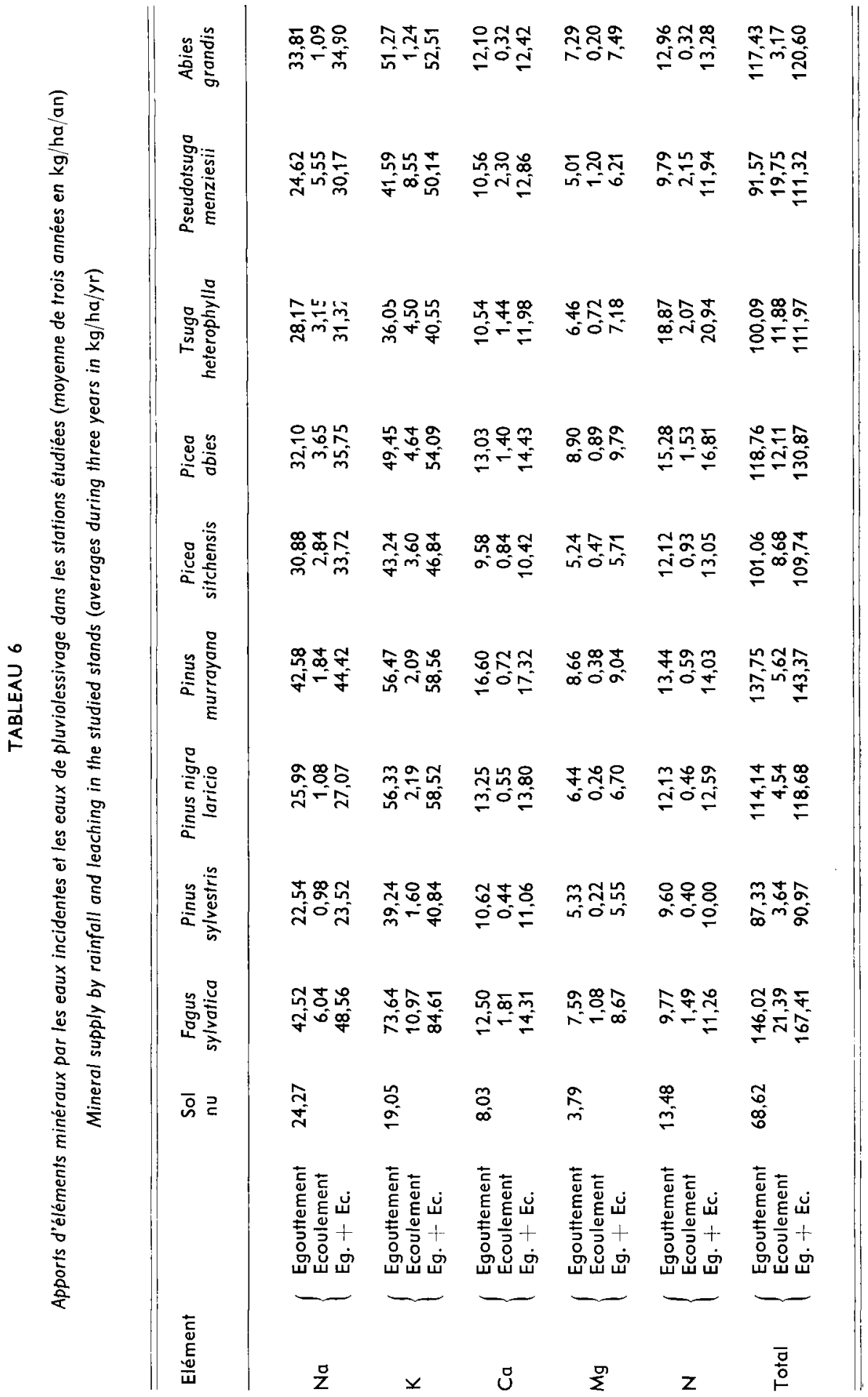


ef Pseudotsuga menziesii et inférieurs sous peuplements de Picea sitchensis, Picea abies, Tsuga heterophylla; sous Abies grandis ils sont du même ordre de grandeur. ha/an.

Le magnésium est apporté en plus petite quantité et ne représente que 5 à $9 \mathrm{~kg} /$

Si nous comparons nos valeurs aux données citées par divers auteurs (tabl. 7), nous remarquons que Denaeyer-de Smet (1966) trouve sous Fagus sylvatica des apports de sodium ef de potassium inférieurs aux nôtres, alors que les apports de calcium sont supérieurs. Ceci s'explique aisément d'une part par la situation géographique et d'autre part par la nature du substrat (calcaires compacts) de la station étudiée par cet auteur. Les résulłałs présentés pour cette même espèce par Nihlgard (1970) sont également inférieurs aux nôtres surtout en ce qui concerne le sodium, le potassium et le magnésium.

TABLEAU 7

Apports d'élémenis minéraux par les eaux de pluviolessivage (d'après les données bibliographiques). Résultats en $\mathrm{kg} / \mathrm{ha} / \mathrm{an}$

Mineral supply by leaching (bibliographical data) in $\mathrm{kg} / \mathrm{ha} / \mathrm{yr}$

\begin{tabular}{|c|c|c|c|c|c|c|c|c|c|}
\hline \multirow{4}{*}{$\begin{array}{l}\text { Elément } \\
\mathrm{Na}\end{array}$} & \multirow[b]{4}{*}{$\begin{array}{l}\text { Egouttement } \\
\text { Ecoulement }\end{array}$} & \multicolumn{8}{|c|}{ Essence et Auteur } \\
\hline & & \multicolumn{2}{|c|}{ Fagus sy/vatica } & \multicolumn{3}{|c|}{ Picea abies } & \multirow{2}{*}{$\begin{array}{c}\text { Abies } \\
\text { grandis }\end{array}$} & \multirow{2}{*}{$\begin{array}{c}\begin{array}{c}\text { Tsugo } \\
\text { hetero- } \\
\text { phyllo }\end{array} \\
\begin{array}{c}\text { adgwick } \\
1959\end{array}\end{array}$} & \multirow{2}{*}{$\begin{array}{l}\text { Pinus } \\
\text { nigra }\end{array}$} \\
\hline & & $\begin{array}{c}\text { Denaeyer-de } \\
\text { Smet } 1966\end{array}$ & $\begin{array}{c}\text { Nihlgard } \\
1970\end{array}$ & $\begin{array}{c}\text { Madgwick } \\
1959\end{array}$ & $\begin{array}{c}\text { Aussenac } \\
1972\end{array}$ & $\begin{array}{c}\text { Nihlgard } \\
1970\end{array}$ & & & \\
\hline & & $\begin{array}{c}19,9 \\
-\end{array}$ & $\begin{array}{r}13,7 \\
2,0\end{array}$ & $\begin{array}{c}27,7 \\
-\end{array}$ & - & $\begin{array}{r}22,6 \\
3,6\end{array}$ & $\begin{array}{c}44,6 \\
-\end{array}$ & $\stackrel{46,0}{-}$ & $\stackrel{24,2}{-}$ \\
\hline K & $\begin{array}{l}\text { Egouttement } \\
\text { Ecoulement }\end{array}$ & 11,0 & $\begin{array}{l}9,9 \\
3,2\end{array}$ & $\begin{array}{c}27.8 \\
-\end{array}$ & $\begin{array}{r}21,37 \\
2,12\end{array}$ & $\begin{array}{r}22,6 \\
4,5\end{array}$ & $\underline{18,5}$ & $\underline{16,5}$ & $\underline{18,7}$ \\
\hline $\mathrm{Ca}$ & $\begin{array}{l}\text { Egouttement } \\
\text { Ecoulement }\end{array}$ & $\frac{22,2}{-}$ & $\begin{array}{l}9,0 \\
1,1\end{array}$ & 16,3 & $\begin{array}{r}12,25 \\
1,21\end{array}$ & $\begin{array}{r}14,7 \\
2,7\end{array}$ & $\underline{31,1}$ & 29,3 & 18.5 \\
\hline $\mathrm{Mg}$ & $\begin{array}{l}\text { Egouttement } \\
\text { Ecoulement }\end{array}$ & - & $\begin{array}{l}3,0 \\
0,4\end{array}$ & $\underline{7,9}$ & $\begin{array}{l}3,04 \\
0,27\end{array}$ & $\begin{array}{l}5,25 \\
0,90\end{array}$ & $\stackrel{9.5}{-}$ & $\frac{9,9}{-}$ & $\stackrel{6.6}{-}$ \\
\hline$N$ & $\begin{array}{l}\text { Egouttement } \\
\text { Ecoulement }\end{array}$ & $\overline{-}$ & $\begin{array}{l}8,5 \\
0,4\end{array}$ & - & $\begin{array}{r}25,75 \\
1,42\end{array}$ & $\begin{array}{r}21,5 \\
2,6\end{array}$ & - & - & - \\
\hline
\end{tabular}

Picea abies a été étudié par divers auteurs dont Madgwick (1959), Nihlgard (1970), Aussenac (1972). Nos résultats sont supérieurs aux leurs pour le sodium, le potassium et le magnésium ; du même ordre de grandeur pour le calcium et inférieurs pour l'azote.

Pour Abies grandis et Tsuga heterophylla, Madgwick (1959) trouve des quantités plus importantes de sodium, calcium et magnésium ; seules nos valeurs de potassium sont supérieures. Cet auteur a également étudié un peuplement de Pinus nigra ; ses résultats sont comparables aux nôtres sauf en ce qui concerne le potassium. 
En ce qui concerne les variations mensuelles, l'apport de sodium est nettement plus important pendant la période hivernale. Ce phénomène est beaucoup moins net dans le cas du magnésium et du calcium. Lemée (1974) précise que le calcium est lessivé en plus grande quantité entre octobre et janvier. Pour cet auteur l'entraînement du magnésium est parallèle à celui du calcium et prend des valeurs plus importantes en phase de chute des feuilles et au début de la phase défeuillée. Madgwick et Ovington (1959) signalent une tendance d̀ un maximum automnal et un minimum printanier pour les apports de calcium.

Pour le potassium nous avons observé des apports irréguliers et très variables au cours de l'année. Denaeyer-de Smet (1966) pense que «l'apport de potassium peut varier suivant les mois, mais ces variations semblent essentiellement liées à des hauteurs de pluie différentes ».

\subsection{Par les eaux d'écoulement.}

Toutes les données précédenfes ne tiennent compte que des quantités d'éléments minéraux apportées par les eaux d'égouttement; dans les peuplements forestiers l'écoulement des eaux de pluie le long des troncs ne peut être négligé. Plusieurs auteurs se sont intéressés à ce problème (tabl. 3). Nous avons utilisé leurs résultats pour estimer nos pourcentages d'écoulement qui sont les suivants :

- Fagus sylvatica : 10 p. 100 (Horton, 1919 ; Aussenac 1968 et 1975 ; Nihlgard, 1970).

- Pinus sp. : 2 p. 100 (Horton, 1919 ; Aussenac, 1968 ; Rapp et Ibrahim, 1978).

- Picea sp. : 3 p. 100 (Aussenac, 1968 ; Nihlgard, 1970).

- Tsuga heterophylla : 6 p. 100 (Voigt, 1960).

- Pseudotsuga menziesii : 10 p. 100 (Aussenac, 1975 ; Boulangeat, 1978).

- Abies grandis : 1 p. 100 (Aussenac, 1968).

Les valeurs des concentrations des eaux d'égouttement ont été utilisées pour calculer l'apport d'éléments minéraux par les eaux d'écoulement tout en sachant que leurs compositions minérales ne sont pas extactement les mêmes.

L'apport d'éléments minéraux par les eaux d'écoulement (tabl. 6) atteint près de $21,5 \mathrm{~kg} / \mathrm{ha} /$ an sous Fagus sylvatica (cette valeur élevée est surtout due à un apport important de potassium) ; sous les Conifères, il varie de $3,5 \mathrm{~kg} / \mathrm{ha} / \mathrm{an}$ chez Pinus sylvestris à $20 \mathrm{~kg} / \mathrm{ha} / \mathrm{an}$ chez Pseudotsuga menziesii.

\section{4. - Les apports d'éléments minéraux par les litières et les pluviolessivats}

Les apports totaux d'éléments minéraux à la surface du sol pour chaque peuplement, par l'intermédiaire des litières et des pluviolessivats sont reportés dans le tableau 8, les données concernant les litières provenant de travaux précédents (Gloaguen et Touffet, 1974 et 1976).

Pour foutes les espèces, le sodium et le potassium sont apportés en quantités nettement plus grandes par les pluviolessivats, contrairement à l'azote bien plus abondant dans les litières qui sont également plus riches en calcium (sauf pour Tsuga heterophylla). Quant au magnésium les résultats obtenus sont souvent voisins, leur importance relative variant avec les espèces. 


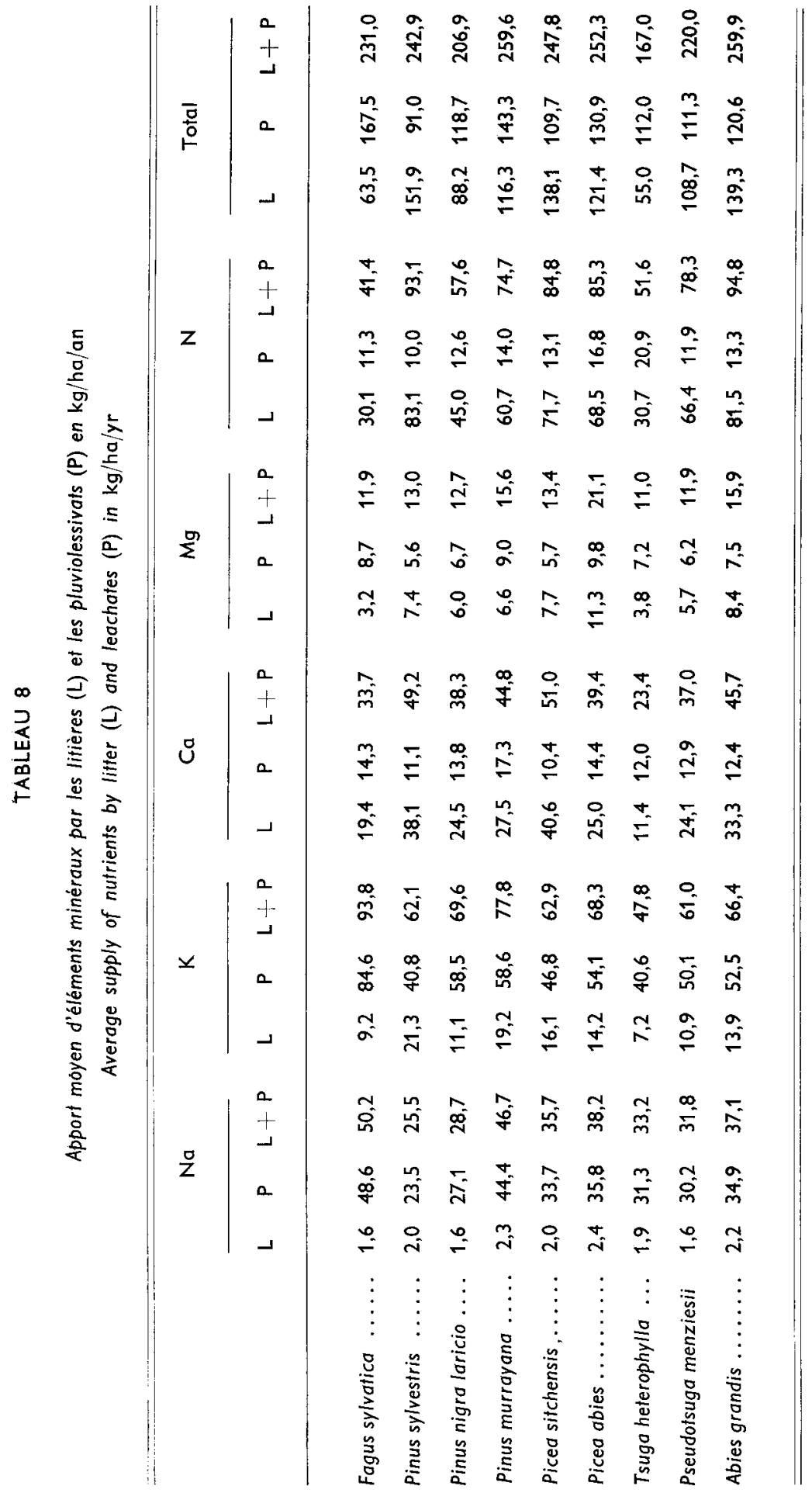


Les apports totaux par les litières sont nettement plus importants pour Pinus sylvestris, Picea sitchensis et Abies grandis, voisins pour Picea abies et Pseudotsuga menziesii, ef bien plus faibles que par les pluviolessivats pour les autres espèces.

Si l'on excepte Tsuga heterophylla pour laquelle l'apport global n'est que de $167 \mathrm{~kg} / \mathrm{ha} / \mathrm{an}$, pour toutes les autres espèces la surface du sol reçoit plus de $200 \mathrm{~kg} / \mathrm{ha}$ / an. C'est Abies grandis et Pinus murrayana qui ont la valeur la plus élevée avec $260 \mathrm{~kg}$ / ha/an, Fagus sylvatica occupant une position moyenne.

\section{IV. - Conclusion}

Cette étude sur l'interception des précipitations et les apports d'éléments minéraux par les eaux de pluie et les pluviolessivats nous a permis de préciser, pour une hêtraie atlantique et quelques peuplements de résineux, les points suivants :

1) L'interception des eaux de pluie par les Conifères est supérieure à celle du Hêtre pour une même surface terrière. Pour la hêtraie, l'interception, indépendante de l'âge du peuplement, dépend plutôt de sa structure.

2) Sous climat atlantique, le taux d'incerception varie beaucoup au cours de l'année. Pour le Hêtre il n'y a pas de relation entre ce taux et les saisons alors que pour les Conifères il est généralement plus faible au printemps el en été.

3) Les eaux de pluie incidentes sont plus riches en sodium pendant l'hiver. Pour les autres éléments minéraux nous n'avons pas mis en évidence de variations saisonnières.

4) Les eaux de pluviolessivage sont toujours plus concentrées que les eaux incidentes. Cette concentration varie selon les éléments minéraux et les essences. Les eaux recueillies sous Pinus sylvestris sont peu concentrées, au contraire sous Picea abies elles ont toujours des teneurs très élevées; sous la hêtraie les concentrations varient selon l'élément, assez forfes pour le sodium et le potassium, elles sont faibles pour le calcium.

5) Les eaux de pluie incidentes apportent $68,5 \mathrm{~kg} / \mathrm{ha} /$ an d'éléments minéraux. Les apports d'éléments par les eaux d'égouttement sont plus importantes sous la hêtraie $(146 \mathrm{~kg} / \mathrm{ha} / \mathrm{an}$ ) que sous les peuplements de résineux (de $87,5 \mathrm{~kg} / \mathrm{ha} / \mathrm{an}$ pour Pinus sylvestris à $138 \mathrm{~kg} / \mathrm{ha} /$ an pour Pinus murrayana). Les quantités d'éléments minéraux apportés par écoulement le long des troncs, estimées à $21 \mathrm{~kg} / \mathrm{ha} / \mathrm{an}$ pour Fagus sylvatica sont toujours inférieures pour les résineux (de 3,5 à $20 \mathrm{~kg} / \mathrm{ha} / \mathrm{an}$ ).

En conclusion, la surface du sol reçoit sous la hêtraie une plus grande quantité d'eau et d'éléments nutritifs pluviolessivés que sous les peuplements de résineux. Par contre l'apport par les litières des jeunes résineux est plus important (sauf pour Tsuga heterophylla) et compense le déficit.

Reçu pour publication en avril 1979.

\section{Summary}

Interception of precipitations and mineral supply to the soil by rainfall and leaching in an altantic beech forest and in some coniferous stands in brittany (France)

The interception of precipitations and mineral supply to the soil by rainfall and leaching have been assessed in eight coniferous stands and in an old beech forest during 1973, 1974 and 1975. 
The study on interception was carried out in 1976 and 1977 on seven development stages of a beech forest. This study on precipitation interception and on mineral supply to the soil by rainfall and leaching gave the following results :

1) Interception of rainfall by conifers is higher than that of beech for the same basal area. For the beech forest, the interception though independant of stand age is rather dependant on stand structure.

2) Under an allantic climate, the ratio of interception varies considerably during the year. For beech there is no relation between this ratio and the season, whereas for conifers it is generally lower during spring and summer.

3) In winter, rainfall contains a higher level of $\mathrm{Na}$. For other mineral elements no seasonal variations were observed.

4) The throughfall is always more concentrated than rainfall. This concentration varies according to mineral elements and species. Water sampled under Pinus sylvestris is little concentrated whereas under Picea abies it always has a very high content in mineral elements. Under a beech forest, concentrations vary according to the element considered : fairly high for $\mathrm{Na}$ and $\mathrm{K}$, low for $\mathrm{Ca}$.

5) Rainfall supplies $68,5 \mathrm{~kg} / \mathrm{ha} / \mathrm{yr}$ of mineral elements. Mineral supply by throughfall is higher under a beech forest $(146 \mathrm{~kg} / \mathrm{ha} / \mathrm{yr}$ ) than under coniferous stands (from $87,5 \mathrm{~kg} / \mathrm{ha} / \mathrm{yr}$ for Pinus sylvestris to $138 \mathrm{~kg} / \mathrm{ha} / \mathrm{yr}$ for Pinus murrayana). The amounts of mineral elements supplied by stemflow, estimated at $21 \mathrm{~kg} / \mathrm{ha} / \mathrm{yr}$ for Fagus silvatica are always lower for conifers (from 3,5 to $20 \mathrm{~kg} / \mathrm{ga} / \mathrm{yr}$ ).

In conclusion, the soil surface under a beech forest receives a higher amount of water and leached nutrients than under coniferous stands. On the other hand, supply from the litter of young conifers is higher (except for Tsuga heterophylla) and compensates this deficit.

\section{Références bibliographiques}

AUSSENAC G., 1968. Interception des précipitations par le couvert forestier. Ann. Sci. forest., 25, $135-156$.

AUSSENAC G., 1975. Couverts forestiers et facteurs du climat : leurs interactions, conséquences écophysiologiques chez quelques résineux. Thèse Nancy, $234 \mathrm{p}$.

AUSSENAC G., BONNEAU M. ef LE TACON F., 1972. Restifution des éléments minéraux au sol par l'intermédiaire de la litière et des précipifations dans quatre peuplements forestiers de l'Est de la France. Ecol. Plant., 7, 1-21.

BOULANGEAT C., 1978. Influence de la forêt sur le cycle de l'eau. Etude comparative de l'interception et de l'évapotranspiration d'un peuplement feuillu (Fagus silvatica L.) et de quatre peuplements de Douglas (Pseudotsugo menziesii Mirb.). Station de sylviculture et de production, Nancy, Document 78/03, 44 p. + annexes.

CARLISLE A., BROWN A. H. F. et WHITE E. J., 1966. The organic matter and nutrient elements in the precipitation beneath a sessile oak (Quercus petraea) canopy. J. Ecol., 54, 87-98.

CARLISLE A., BROWN A. H. F. et WHITE E. J., 1967. The nutrient content of tree stem flow and ground flora litter and leachates in a sessile oak (Quercus petraea) woodland. J. Ecol., 55, 615-627.

DENAEYER-DE SMET S., 1966. Bilan annuel des apports d'éléments minéraux par les eaux de précipitation sous couvert forestier dans la forêt mélangée caducifoliée de Blaimont (VirellesChimay). Bull. Soc. roy. Bot. Belg., 99, 345-375.

ESTEOULE J., GUYADER J. et TOUFFET J., 1971. Les sols de la forêt de Villecartier. Bull. Ass. fr. Ef. Sol, 2, 29-46.

FORGEARD F., GLOAGUEN J. C. et TOUFFET J., 1979. Interception des précipifations et apport au sol d'éléments minéraux par les eaux de pluie et les pluviolessivats dans quelques landes armoricaines. Bull. d'Ecologie, 10, 29-41.

GLOAGUEN J. C. et TOUFFET J., 1974. Production de litière et apport au sol d'éléments minéraux dans une hêtraie atlantique. Ecol. Plant., 9, 11-28.

GLOAGUEN J. C. et TOUFFET J., 1976. Production de litière et apport au sol d'éléments minéraux dans quelques peuplements résineux de Bretagne. Ann. Sci. forest., 33, 87-107.

GRATANI L. et BRUNO F., 1974. Apporto di elementi minerali all' ecosistema faggeta (Terminillo) da parte delle acque di precipitazione : comparazione con lo stemflow ed il throughfall. Ann. di Bot., 33, 151-165.

HELVEY J. D. ef PATRIC J. H., 1965. Canopy and litter interception of rainfall by Hardwoods of Eastern United States. Woter Resources Res., 1, 193-206.

HOOVER M. D., 1953. Interception of rainfall in a young loblolly pine plantation. U. S. Forest Service Southeastern Forest Exp. St., 21, 13 p.

HORTON R. E., 1919. Rainfall interception. Monthly weother review, 47, 608-623.

LAUSBERG T., 1935. Quantitative untersuchungen uber die kutikulare. Exkretion des Laubblattes. Jb. Wiss. Bot., 81, 769-806. 
LAWSON E. R., 1967. Throughfall and stemflow in a Pine-Hardwood Stand in the Ouachifa Mountain of Arkansas. Water Resources Res., 3, 731-735.

LEMEE G., 1974. Recherches sur les écosystèmes des réserves biologiques de la forêt de Fontainebleau. IV. - Entrées d'éléments minéraux par les précipitations et transfert au sol par le pluviolessivage. Fcol. Plant., 9, 187-200.

LEONARD R. E., 1966. Mathematical theory of interception. International Symposium of Forest Hydrology. Pennsylvania State University, Aug.-Sept. 1965, Pergamon Press, Oxford New-York, $131-136$.

MADGWIK M. A. I. et OVINGTON J. D., 1959. The chemical composition of precipitation in adjacent forest and open plots. Forestry, 32, 14-22.

MAYER R. et ULRICH B., 1974. Conclusions on the filfering action of forests from ecosystem analysis. G.col. Plant., 9, 157-168.

MILLER R. B., 1963. Plant nutrients in hard beech. III The cycle of nutrients. N. Z. J. Sci., 6, 388-413.

NIHLGARD B., 1970. Precipitation, its chemical composition and effect on soil water in a beech and spruce forest in south Sweden. Oikos, 21, 208-217.

NOIRFALISE A., 1959. Sur l'interception de la pluie par le couvert dans quelques forêts belges. Bull. Soc. roy. For. Belg., octobre 1959, 1-7.

OVINGTON J. D., 1954. A comparison of rainfall in different woodlands. Forestry, 27, 41-53.

RAPP M., 1969. Apport d'éléments minéraux au sol par les eaux de pluviolessivage sous des peuplements de Quercus ilex L., Quercus lanuginosa Lamk. ef Pinus halepensis Mill. CEcol. Plant., 4, 71-92.

RAPP M. et ROMANE F., 1968. Contribution à l'étude du bilan de l'eau dans les écosystèmes méditerranéens. 1. - Egouttement des précipitations sous des peuplements de Quercus ilex $L$. et de Pinus holepensis Mill. Gcol. Plant., 3, 271-284.

RAPP M. et COLE D. W., 1973. Evolution des éléments minéraux dissous dans les précipitations ef les percolats du sol à travers un écosystème forestier. Ann. Sci. forest., 30, 175-190.

RAPP M. et IBRAHIM M., 1978. Egouttement, écoulement et interception des précipitations par un peuplement de Pinus pinea L. Ecol. Plant., 13, 321-330.

ROGERSON T. L. et BYRNES W. R., 1968. Net rainfall under Hardwoods and Red Pine in Central Pennsylvania. Water Resources Res., 4, 55-57.

SCHNOK G. et GALOUX A., 1967. Recherches sur l'écosystème forêt. Série B : La chênaie mélangée calcicole de Virelles-Blaimont. Contribution $n^{0} 8$ : Réception des précipitations ef égouttement. Bull. Inst. roy. Sc. Nat. Belg., 43, 33, 1-30.

SKAU C. M., 1964. Interception, throughfall and stemflow in Utah and Alligator Juniper Cover types of Northern Arizona. Forest Science, 10, 283-287.

SZABO M. et CSORTOS C., 1975. A study of the nutrient content of canopy throughfall in an oak forest (Quercetum petraeae-cerris) measured for one year. Act. Bot. Acad. Sc. Hung., 21, 419-432.

TOUFFET J., 1964. Aperçu bryologique sur la forêt de Villecartier (Ille-et-Vilaine) : les Sphaignes. Bull. Soc. Sci. Brefagne, 39, 69-75.

TOUFFET J., 1971. Contribution à l'étude de la bryoflore de la forêt de Villecartier (llle-et-Vilaine). Botanica Rhedonica, sér. A, 11, 189-222.

VOIGT G. K., 1960. Distribution of rainfall under forest stands. Forest Sci., 6, 2-10.

WHITE E. J. et CARLISLE A., 1968. The interception of rainfall by mixed deciduous woodland. Quart. J. For., 62, 310-320.

WOLAVER T. G. ef LIETH H., 1973. Modeling the precipitation chemistry across the U. S. EDV in Medizin und Biologie, 4, 74-87. 